\title{
DOPPLER MEASUREMENT OF DIFFERENTIAL CROSS SECTIONS IN CROSSED BEAM EXPERIMENTS: DEPENDENCE OF THE DETECTION SENSITIVITY WITH THE PRODUCT VELOCITY
}

\author{
N. BILLY, B. GIRARD, G. GOUÉDARD and J. VIGUÉ \\ Laboratoire de Spectroscopie Hertzienne de l'E.N.S. $\dagger$ \\ 24, rue Lhomond-75231 Paris Cédex 05-France
}

(Received 15 November, 1989; in final form 15 December, 1989)

\begin{abstract}
Differential cross sections can be measured as a function of the internal state of a reaction product thanks to the analysis of the Doppler profile of the laser induced fluorescence detection line. This analysis is complicated by two effects: first, the LIF signal intensity depends on the interaction time of the molecule with the laser, and this time depends on the scattering angle, second, the angular and velocity distributions of the beams have non negligible widths. We present here a treatment of these effects in the case of the $F+I_{2}$ reaction for which we have measured the differential cross section by this technique. The same formalism is also applied to the deduction of the rovibrational distribution of the products from the relative intensities of the LIF lines.
\end{abstract}

KEY WORDS: Reactive scattering, differential cross section, crossed molecular beams, laser-induced fluorescence, Doppler profile.

\section{INTRODUCTION}

Doppler measurement of the velocity of a collision product can be used to measure the differential cross section of a reaction as a function of the internal state of the detected product. ${ }^{1}$ This very powerful technique has been used many times in photodissociation experiments ${ }^{2}$ and only in a few collision experiments. ${ }^{3}$ We have performed such an experiment for the $F+I_{2}$ reaction and in order to analyse these data, it appears necessary to model carefully the relation between the differential cross section and the Doppler profile of the laser induced fluorescence signal. Two main effects must be taken into account:

-as soon as the product recoil velocity in the center of mass frame is not small with respect to the velocity of this frame, the detection sensitivity depends noticeably on the direction of the recoil velocity;

-the experiment is not an ideal one, in the sense that the beams have non negligible velocity and angular spreads, and this induces various averaging effects.

† Associated with Ecole Normale Superieure, Universtité P. et M. Curie and C.N.R.S. (UA 18). The authors are also belong to GRECO 87 (C.N.R.S.) "Dynamique des Réactions Moléculaires." 
The goal of the present paper is to set-up the mathematical formalism necessary to represent as exactly as possible these two effects in the analysis of the experimental data. This formalism is used to establish the relation between the differential cross section and the Doppler profile as well as the total intensity of the LIF signals.

\section{DOPPLER PROFILE OF THE LASER INDUCED FLUORESCENCE SIGNAL IN AN IDEAL EXPERIMENT}

We analyse here the LIF signal given by a perfect crossed beam experiment, perfect in the sense that we do not consider here velocity distribution or angular spread of the beams. So we only deal with the detection sensitivity versus the direction of the recoil velocity. Previously a special attention is given to the nature of the measured signal.

\section{II.1 Principles}

We will particularize immediately to the case of the $F+I_{2}$ reaction but, except where noted, this will be done without loss of generality, as long as one considers only reaction between an atom and a diatomic molecule. So we consider the following reaction:

$$
F+I_{2} \longrightarrow I F+I
$$

which is endothermic: $\Delta H_{0}=-1.23 \mathrm{eV} /$ molecule. The geometrical arrangement is represented on Figure 1: two molecular beams cross at right angle and we assume

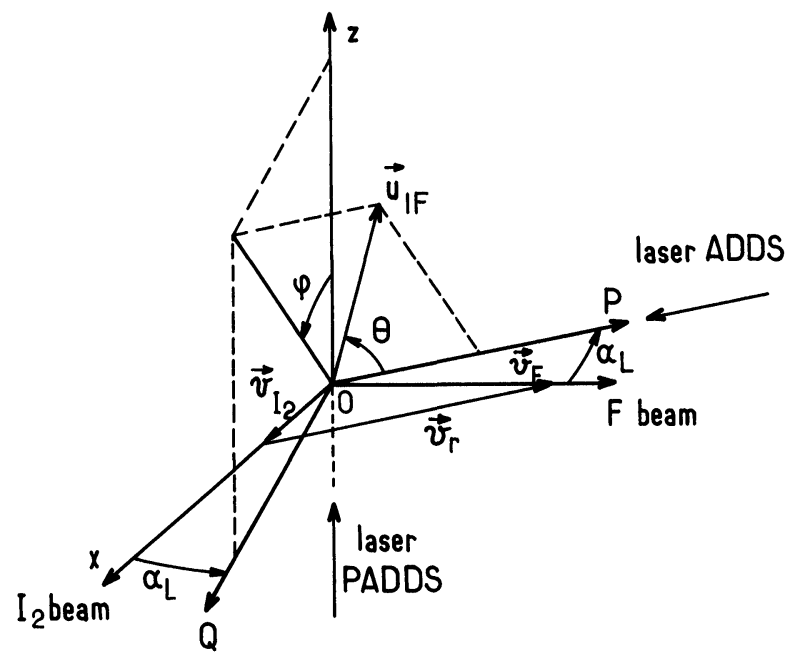

Figure 1 Geometrical arrangement: the $I_{2}$ beam (along the $O x$ axis) and the $F$ beam (along the $O y$ axis) cross at right angle. The OP axis is along the relative velocity $v_{r}=v_{F}-v_{I_{2}}$ and $O Q$ is the direction perpendicular to OP in the Oxy plane. In ADDS geometry, the laser is colinear to the relative velocity, i.e. along OP and, in PADDS geometry, it is perpendicular to the two beams, i.e., along Oz. In the centre of mass frame, the IF product velocity $\mathrm{u}_{I F}$ is described by its spherical coordinates $\mathrm{u}_{I F}, \theta, \varphi$ relative to the frame $\mathrm{OzQP}$. 
here that their velocity distributions as well as their angular distributions have negligible widths. Accordingly, the centre of mass velocity $\mathbf{v}_{G}$ and the relative velocity $\mathbf{v}_{r}$ do not present any dispersion:

$$
\begin{aligned}
\mathbf{v}_{G} & =\left(m_{I_{2}} \mathbf{v}_{I_{2}}+m_{F} \mathbf{v}_{F}\right) / M \\
\mathbf{v}_{r} & =\mathbf{v}_{F}-\mathbf{v}_{I_{2}}
\end{aligned}
$$

with obvious notations $\left(M=m_{F}+m_{I_{2}}\right)$.

The available energy for the products, $E_{a v}$, is the sum of the reaction endothermicity and of the collision kinetic energy:

$$
E_{a v}=-\Delta H_{0}+1 / 2 \mu v_{r}^{2}
$$

where $\mu$ is the reduced mass of the reactants $\left(\mu=m_{I_{2}} m_{F} / M\right)$. We have neglected here any internal energy of the reactants. Conservation of energy is not sufficient, by itself, to define the relative velocity of the products, as both products can be excited (the iodine atom can be produced in its ${ }^{2} P_{1 / 2}$ excited fine structure state, but this is a very minor branch of the reaction ${ }^{4}$ ). However for IF levels with sufficient internal energy (in our conditions $v>6$ ) the iodine atom is necessarily produced in its ground state and the final relative velocity $\mathbf{v}_{r}^{\prime}$ has a modulus given by:

$$
1 / 2 \mu^{\prime} \mathbf{v}_{r}^{\prime 2}=E_{a v}-E(v, J)
$$

where $E(v, J)$ is the internal energy of IF in its $v, J$ rovibrational level and $\mu^{\prime}$ is the reduced mass of the products $\left(\mu^{\prime}=m_{I} m_{I F} / M\right)$. Then the velocity $\mathbf{u}_{I F}$ of IF in the level $v, J$, in the centre of mass frame, has a modulus given by:

$$
u_{I F}=\frac{m_{I}}{M} \mathrm{v}_{r}^{\prime}
$$

The collision problem, in the centre of mass frame, presents a cylindrical symmetry around the reactant relative velocity vector $\mathbf{v}_{r}$. Therefore, the velocity $\mathbf{u}_{I F}$, which is distributed on a sphere, is equally probable on each circle of axis OP (OP is parallel to $\mathbf{v}_{r}$, and is taken as the polar axis). To take advantage of this symmetry, one must shine the laser along OP. If $\omega_{0}$ is the angular frequency at resonance for the LIF transition, the Doppler shifted resonance frequency is given by:

$$
\omega_{r}=\omega_{0}\left[1-\frac{v_{G P}+u_{I F} \cos \theta}{c}\right]
$$

Here $\mathrm{v}_{\mathrm{GP}}$ is the projection of $\mathrm{v}_{G}$ on the OP axis, and $u_{I F}, \theta, \varphi$ are the spherical coordinates of the vector $\mathbf{u}_{I F}$. The - sign appears when the laser propagates in a direction opposite to $\mathbf{v}_{r}$, as in Figure 1 . Because $\omega_{r}$ depends only on $\theta$ (and not on $\varphi$ ), this arrangement, named ADDS, ${ }^{5}$ allows a direct measurement of the differential cross section $[\mathrm{d} \sigma / \mathrm{d} \Omega](\theta)$ in the centre of mass frame. But because $\theta$ appears only through its cosine, the angular resolution of this measurement is very poor near $\theta=0$ and $\pi$. This can be remedied by using a geometrical arrangement in which the laser 
beam is along the axis $O z$, perpendicular to the plane of the molecular beams. In this case, the resonance frequency is given by:

$$
\omega_{r}=\omega_{0}\left[1+\frac{u_{I F} \sin \theta \cos \varphi}{c}\right]
$$

The angular resolution near $\theta=0$ and $\pi$ is then good, but in this arrangement (named PADDS), the signal at frequency $\omega_{r}$ corresponds to a range of $\theta$ values (and not to a single one, as for ADDS). Moreover the signal is only sensitive to the sum [d $\sigma / \mathrm{d} \Omega$ ] $(\theta)+[\mathrm{d} \sigma / \mathrm{d} \Omega](\pi-\theta)$, i.e., one cannot distinguish backward and forward scattering. ${ }^{5}$

\section{II.2 The Measured Signal}

Let us now consider the laser induced fluorescence signal. We assume that the laser is a c.w. single frequency laser with a long coherence time. We have already discussed in details the saturation effects in this case. ${ }^{6}$

The LIF signal, i.e., the number of photons emitted per unit time for a laser frequency $\omega_{L}$ is:

$$
s\left(\omega_{L}-\omega_{0}\right)=\int x\left(\omega_{L}-\omega_{r}\right) \mathrm{d}^{2} \dot{N}
$$

where $x\left(\omega_{L}-\omega_{r}\right)$ is the number of fluorescence photons emitted by one molecule, for a laser detuning $\omega_{L}-\omega_{r}$, and $\mathrm{d}^{2} N$ represents the number of molecules produced per unit time in the $(\theta, \varphi)$ direction in the centre of mass frame. Obviously we have:

$$
\mathrm{d}^{2} \stackrel{\circ}{N}=n_{I_{2}} n_{F} \mathrm{v}_{\mathbf{r}} \frac{\mathrm{d} \sigma}{\mathrm{d} \Omega} \cdot \mathrm{V} \sin \theta \mathrm{d} \theta \mathrm{d} \varphi
$$

Here $n_{I_{2}}$ and $n_{F}$ are the densities of the two beams, $v$ is the collision volume and $(\mathrm{d} \sigma / \mathrm{d} \Omega)$ is the reaction differential cross section in the centre of mass frame.

Starting from Eq. (9), we discuss now two problems: first, the ultimate velocity resolution of such an experiment and second, the variation of the unimolecular signal with the interaction time between the IF product and the probe laser.

\section{Velocity Resolution}

The $x\left(\omega_{L}-\omega_{r}\right)$ function has been studied in Ref. (6), especially under resonant conditions. This quantity is resonant for $\omega_{L}=\omega_{r}$. The resonance width $\partial \omega$ is proportional to the ultimate velocity resolution of the Doppler profile. In the absence of saturation, it is equal to the natural width $\Gamma$ of the excited state, and this corresponds to a velocity resolution $\partial \mathrm{v}=\left(\Gamma / \omega_{0}\right) c$ (of the order of $0.02 \mathrm{~m} / \mathrm{s}$ for the IF $\mathrm{B}-\mathrm{X}$ transition). Saturation is due to power broadening of the resonance. We consider here only the saturation case which applies to our experiment:

$$
\omega_{1} \tau \gg 1
$$

where $\omega_{1}$ is the Rabi frequency of the transition, and $\tau$ the interaction time with the 
laser field (supposed to be constant inside the beam and zero outside). In that case, the saturation width is:

$$
\begin{gathered}
\partial \omega=\omega_{1} \text { for } \Gamma \tau \ll 1 \\
\partial \omega=\omega_{1} \sqrt{\Gamma \tau} \text { for } \Gamma \tau \gg 1
\end{gathered}
$$

In our experimental conditions, we have $\Gamma \tau \sim 1$ and $\omega_{1}$ is in the range $10^{7}-10^{8} \mathrm{~s}^{-1}$. The corresponding velocity resolution $\partial \mathrm{v}=\left(\omega_{1} / \omega_{0}\right) c$ remains very good $(\partial \mathrm{v} \simeq$ $10 \mathrm{~m} / \mathrm{s})$.

\section{Variation of the Individual Signal with the Interaction Time}

Using the same assumption as in Ref. (6), i.e., the resonance width is smaller than any structure of $(\mathrm{d} \sigma / \mathrm{d} \Omega)$, expressed in frequency unit, we get:

$$
x\left(\omega_{L}-\omega_{r}\right)=I \partial\left(\omega_{L}-\omega_{r}\right)
$$

with $I=\int_{-\infty}^{+\infty} x(\omega) \mathrm{d} \omega . I$ appears to be proportional to the mean LIF signal produced by one molecule at resonance. If $\omega_{1} \tau \gg 1, I$ is given by: ${ }^{7}$

$$
I=\pi \omega_{1} \frac{\Gamma_{\mathrm{rad}} \tau}{2} \exp \left(-\frac{\Gamma \tau}{2}\right)\left[I_{0}\left(\frac{\Gamma \tau}{2}\right)+I_{1}\left(\frac{\Gamma \tau}{2}\right)\right]
$$

Here $\Gamma_{\text {rad }}$ is the radiative decay rate of the excited state (different from $\Gamma$ if this state suffers predissociation), and $I_{0}$ and $I_{1}$ are modified Bessel functions. ${ }^{8} I$ behaves asymptotically like:

$$
\begin{gathered}
I=\frac{\pi}{4} \omega_{1} \Gamma \tau \text { for } \Gamma \tau \ll 1 \\
I=\sqrt{\pi} \omega_{1} \sqrt{\Gamma \tau} \text { for } \Gamma \tau \gg 1
\end{gathered}
$$

(with $\Gamma_{\mathrm{rad}}=\Gamma$ ). If we consider that the experiment is in the first case, the signal is a linear function of the interaction time $\tau$ (and this would be also the case for an incoherent laser in the absence of saturation). In any case, $I$ depends on the direction $(\theta, \varphi)$ of the IF velocity, via the interaction time $\tau$, and the LIF signal is:

$$
s\left(\omega_{L}-\omega_{0}\right)=\int \partial\left(\omega_{L}-\omega_{r}\right) I(\tau) \mathrm{d}^{2} \stackrel{\circ}{N}
$$

\section{II.3 The Doppler Profile}

We first consider here the ADDS geometry. The laser beam is assumed to be centered on the OP axis, and has a radius $r_{L}$. For a very small scattering volume centered in $O$, the interaction time is given by:

$$
\tau(\theta, \varphi)=\frac{r_{L}}{\left[\mathrm{v}_{G Q}^{2}+u_{I F}^{2} \sin ^{2} \theta+2 \mathrm{v}_{G Q} u_{I F} \sin \theta \cos \varphi\right]^{1 / 2}}
$$


where $\mathbf{v}_{G Q}$ is the component of $\mathbf{v}_{G}$ on the axis OQ. In the two following limiting cases, $\tau$ is dependent of $\varphi$ :

$$
\begin{array}{ll}
u_{I F} \gg \mathrm{v}_{G Q} \quad \tau=\frac{r_{L}}{u_{I F} \sin \theta} \\
u_{I F} \ll \mathrm{v}_{G Q} \quad \tau=\frac{r_{L}}{\mathrm{v}_{G Q}}
\end{array}
$$

and the calculation of the $\varphi$ average of $\tau$ is trivial. We see that in general, the $\varphi$ average of $\tau$ will present a strong dependence on $\theta$, and the detection sensitivity will have the same dependence. For example, in the limiting case $u_{I F} \gg v_{G Q}$ (and for $\Gamma \tau \ll$ 1 , i.e., $I \sim \tau)$, the Doppler profile $s\left(\omega_{L}-\omega_{0}\right)$ is proportional to:

$$
s\left(\omega_{L}-\omega_{0}\right) \sim \frac{\mathrm{d} \sigma}{\mathrm{d} \Omega}(\theta) \frac{1}{\sin \theta}
$$

where $\cos \theta=\frac{\omega_{L}-\omega_{0}}{\omega_{0}} \frac{c}{u_{I F}}$.

The same effect acts also in the PADDS geometry. Now the laser beam is assumed to be centered on the $\mathrm{OZ}$ axis. The same kind of hypothesis leads, in the limiting case $u_{I F} \gg v_{G}$, to a Doppler profile proportional to:

$$
s\left(\omega_{L}-\omega_{0}\right) \sim \frac{1}{\sqrt{1}-D^{2}} \int_{0}^{\sqrt{1-D^{2}}}\left[\frac{\mathrm{d} \sigma}{\mathrm{d} \Omega}(\theta)+\frac{\mathrm{d} \sigma}{\mathrm{d} \Omega}(\pi-\theta)\right] \frac{\mathrm{d} \cos \theta}{\left(1-D^{2}-\cos ^{2} \theta\right)^{1 / 2}}
$$

where $D=\frac{\omega_{L}-\omega_{0}}{\omega_{0}} \frac{c}{u_{I F}}$

\section{MODELISATION OF OUR EXPERIMENT}

\section{III.1 Averaging Effects}

In addition to the effect discussed above, we are going to take into account the averaging effects introduced by the finite widths of the angular and velocity distributions of the molecular beams, as well as the internal state distribution of the Iodine beam.

We represented each molecular beam as a perfect molecular flow emitted by a point source $S$. The density at a point $M$ near the scattering center $\mathrm{O}$ is given by:

$$
n(M, \mathrm{v}) \mathrm{dv}=n_{0}\left(\frac{S O}{S M}\right)^{2} p(\mathrm{v}) \mathrm{dv}
$$

where $n_{0}$ is the total density at $O$. The velocity vector $\mathbf{v}$ is assumed to be parallel to the 
vector SM and the distribution of its modulus $v$ is given by the formula usually accepted for supersonic molecular beams: ${ }^{9}$

$$
p(\mathrm{v})=C \mathrm{v}^{2} \exp -\left(\frac{\mathrm{v}-u}{\alpha}\right)^{2}
$$

( $C$ is the normalization constant, $u$ and $\alpha$ are defined in Ref. (9) and can be measured by the time of flight technique). Each beam is collimated (by a circular orifice for the Fluorine beam, by a long slit for the Iodine beam) and we assume that the density vanishes out of the volume defined by $S$ and the collimator. We neglect the possible angular dependence ${ }^{10}$ of the density inside this volume; this is a good approximation as the angular width of the collimator of the beam is small.

The internal state distribution of the Iodine molecules is characterized by two temperatures, namely a vibrational one $T_{\nu}$, and a rotational one $T_{r}$. This non equilibrium distribution is well known in supersonic beams and can easily be characterized by spectroscopic techniques for Iodine. ${ }^{11}$ The probability of occurrence of the $\left(v^{\prime \prime}, J^{\prime \prime}\right)$ rovibrational level is then proportional to:

$$
P\left(v^{\prime \prime}, J^{\prime \prime}\right) \sim\left(2 J^{\prime \prime}+1\right) \exp \left[-\frac{\hbar \omega v^{\prime \prime}}{k T_{v}}-\frac{B J^{\prime \prime}\left(J^{\prime \prime}+1\right)}{k T_{r}}\right]
$$

Ortho-para alternation is here neglected. Hereafter rotation is treated through the continuous variable $x=J^{\prime \prime}\left(J^{\prime \prime}+1\right)$.

The fluorine atom is also distributed on its two fine structure states $\left({ }^{2} P_{3 / 2} E=0\right.$, $\left.{ }^{2} P_{1 / 2} E=404 \mathrm{~cm}^{-1}\right)$, probably with an equilibrium distribution at the oven temperature $(T \simeq 1000 \mathrm{~K})$. Because the potential energy surfaces which correlate to these two states are different, it is not realistic to assume that the two types of collision lead to the same differential cross section. We have chosen her to treat only the collision involving the ${ }^{2} P_{3 / 2}$ state. Neglecting the ${ }^{2} P_{1 / 2}$ contribution to describe our data is supported by the fact that this state represents at most $20 \%$ of the reactants and may be a very good assumption if the corresponding potential energy surface is repulsive or presents a high barrier.

Taking into account these averages, the LIF signal for a laser frequency $\omega_{L}$ becomes:

$$
\begin{aligned}
s\left(\omega_{L}-\omega_{0}\right)= & \sum_{v^{\prime \prime \prime}} \int \partial\left(\omega_{L}-\omega_{r}\right) I(\tau) \\
& \times P\left(v^{\prime \prime}, J^{\prime \prime}\right) n_{I_{2}}\left(M, \mathbf{v}_{\mathbf{I}_{2}}\right) \mathrm{dv}_{\mathrm{I}_{2}} n_{F}\left(M, \mathrm{v}_{\mathrm{F}}\right) \mathrm{dv}_{\mathrm{F}} \mathbf{v}_{\mathbf{r}} \frac{\mathrm{d} \sigma}{\mathrm{d} \Omega}(\theta) \mathrm{d}^{3} \mathrm{~V} \sin \theta \mathrm{d} \theta \mathrm{d} \varphi
\end{aligned}
$$

It appears as a mean over all the trajectories. $\tau$ is the interaction time calculated exactly for each trajectory, defined by the point $M$ where the collision occurs, and the IF velocity $\mathbf{v}_{G}+\mathbf{u}_{I F}$. The initial conditions fix the available energy, and consequently the $u_{I F}$ modulus. The $\partial$ function expresses the condition on the IF velocity along the laser axis which gives the right Doppler shift (as in Eq. 16). 
It should be noticed that the time $\tau$ is not a simple function of the parameters. Namely three different cases occur:

-some trajectories begin out of the laser beam and do not go through it (then $\tau=0$ ),

- some other trajectories, beginning outside the laser beam, go through it,

-and finally some trajectories begin inside the laser beam. For the last two cases, the algebraical expressions of the time $\tau$ are given in the appendix. We consider here that the observation zone is large enough to allow the detection of the fluorescence from each molecule along the whole part of its trajectory inside the laser field.

In fact what we are looking for is not to calculate the LIF signal $s\left(\omega_{L}-\omega_{0}\right)$ from $(\mathrm{d} \sigma / \mathrm{d} \Omega)$, but to invert this relation. This can be done practically by a least square fit for the following reasons:

- The signal at frequency $\omega_{L}$ appears to be a convolution of the differential cross section by a complicated apparatus function defined by Eq. (24).

-In our case, IF B-X transitions present an hyperfine structure ${ }^{12}$ which introduces further complications. The observed signal can be written as:

$$
S\left(\omega_{L}\right)=\sum_{k} \mid \alpha_{k} s\left(\omega_{L}-\omega_{k}\right)
$$

where $\omega_{k}$ is the frequency of the $k^{\text {th }}$ hyperfine component of the transition, and $\alpha_{k}$ its weight factor. The differential cross section, and consequently the signal $s$, must be independent of $k$ if we assume that the hyperfine Hamiltonian is too small to be efficiently coupled during the collision, i.e., if:

$$
\mathscr{H}_{H F S} \cdot t_{\text {coll }} \ll \hbar
$$

This is a well known property in collision theory ${ }^{13}$ and condition (26) should be fulfilled as the estimated duration of the reactive collision $t_{\text {coll }}$ is a few picoseconds ${ }^{14}$ and the order of magnitude of the hyperfine Hamiltonian due to the Iodine nucleus is $\mathscr{H}_{H F S} / \hbar \sim 10^{10} \mathrm{~s}^{-1} .^{15}$

\section{III.2 Signal Simulation}

In order to fit the experimental data, we develop the differential cross section $(\mathrm{d} \sigma / \mathrm{d} \Omega)$ on a polynomial basis in $\cos \theta$; we have chosen to use Legendre polynomials:

$$
\frac{\mathrm{d} \sigma}{\mathrm{d} \Omega}=\sum_{l} \mid \sigma_{l} P_{l}(\cos \theta)
$$

and we calculate the functions $s_{l}\left(\omega_{L}-\omega_{0}\right)$ obtained by replacing $(\mathrm{d} \sigma / \mathrm{d} \Omega)$ by $P_{l}(\cos \theta)$ in Eq. (24).

These integrals are too intricate to be calculated by means other than the Monte-Carlo method. ${ }^{16}$ Figure 2 shows different $s_{l}\left(\omega_{L}-\omega_{0}\right)$ functions for a IF internal energy $E_{I F}=5000 \mathrm{~cm}^{-1}$, which have been computed both for ADDS and PADDS geometries. All the input data are collected in Table 1. Each calculation 

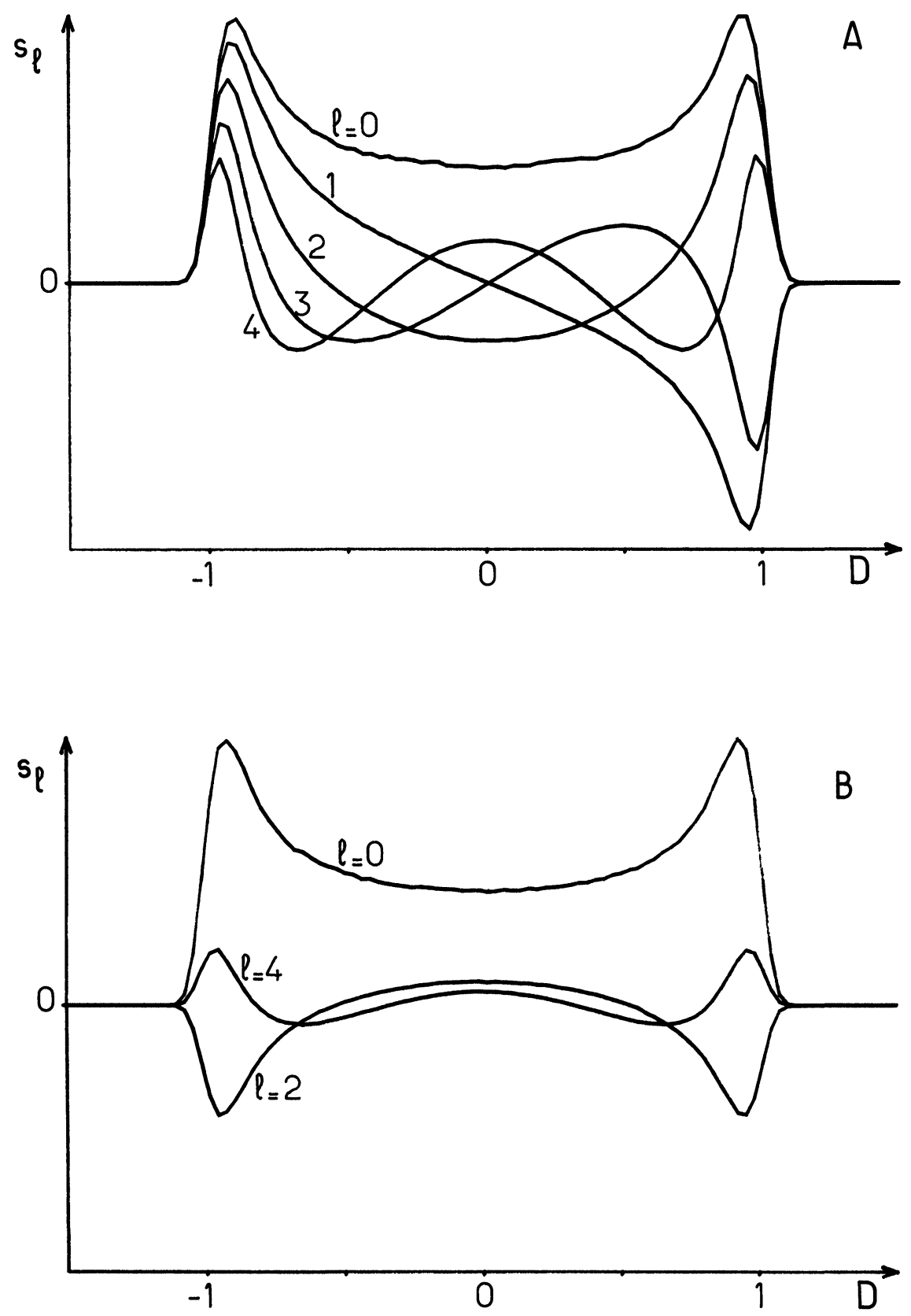

Figure 2 The $s_{l}\left(\omega_{L}-\omega_{0}\right)$ functions with different $l$ values $(0 \leqslant l \leqslant 4)$ obtained for an internal energy $E_{I F}$ $=5000 \mathrm{~cm}^{-1}$, in the ADDS (part a) and PADDS (part b) geometries. $s_{l}$ corresponds to the experimental Doppler profile for a differential cross section proportional to $P_{l}(\cos \theta)$. They are plotted here as a function of the dimensionless parameter $D=\left(\omega_{L}-\omega_{0}\right) /\left(\omega_{0}\right) \times\left(c /\left\langle u_{I F}\right\rangle\right)$ where $\left\langle u_{I F}\right\rangle$ is the quadratic mean of the IF velocity in the centre of mass frame, i.e., the IF velocity corresponding to the mean recoil energy. In PADDS geometry, the $s_{l}$ functions with odd $l$ values average to zero, and have not been plotted here. 
Table 1 Beams and laser parameters used as input data in the calculations presented here.

\begin{tabular}{lccc}
\hline Beam parameters & & F beam & $I_{2}$ beam \\
\hline Distance between the source and & & & \\
the centre of the collision volume & $\mathrm{SO}$ & $45.7 \mathrm{~mm}$ & $14 \mathrm{~mm}$ \\
Angular aperture of the beam & $\operatorname{tg} \alpha$ & $3.710^{-2}$ & $10^{-1}$ \\
Velocity distribution & $u$ & $1,020 \mathrm{~m} / \mathrm{s}$ & $300 \mathrm{~m} / \mathrm{s}$ \\
$\alpha$ & $134 \mathrm{~m} / \mathrm{s}$ & $51 \mathrm{~m} / \mathrm{s}$ \\
Vibrational temperature & $T_{v}$ & & $200 \mathrm{~K}$ \\
Rotational temperature & $T_{r}$ & & $144 \mathrm{~K}$ \\
Laser beam radius & $r_{L}$ & & $1 \mathrm{~mm}$ \\
\hline
\end{tabular}

involves, $2.510^{6}$ trajectories with a non zero interaction time $\tau$ and 100 boxes have been used on the $\omega$ axis.

For ADDS geometry, as expected, $s_{l}$ looks like $P_{l}$. However the experiment has a low sensitivity to the molecules whose velocity is perpendicular to the laser: this induces distortion for $\omega_{L} \simeq \omega_{0}$, i.e. at the center of the Doppler profile. On the contrary, the internal energy distribution and angular averages smooth the two ends of the profile. In PADDS geometry, the $s_{l}$ functions with $l$ odd are quite zero, according to the idea that this arrangement cannot distinguish backward and forward scattering. Because in this geometry, the Doppler shift $\omega_{L}-\omega_{0}$ is not correlated to a single value of $\theta$, even in a perfect experiment, the other curves $s_{l}$ (with $l>0$ ) are not easy to interpret qualitatively, but they are also affected by the effects described above. This can be clearly seen for $l=0$ (corresponding to the case of an isotropic differential cross section).

Figure 3 shows the evolution of the $s_{l=0}$ curves as a function of the IF internal energy, for the two geometries. We verify here that $s_{0}$, which is almost flat for low recoil energy (case 18b), becomes more and more sharply peaked near its extreme values when the recoil energy increases (case 18a). These results show clearly that an analysis of the Doppler profile of the line not taking into account the variation of the interaction time with the velocity direction would give a strongly incorrect differential cross section.

\section{THE INTEGRATED LINE INTENSITY}

The integrated line intensity $S$ is naturally used as a measurement of the total cross section. However the effects discussed in Part III affect also this measurement. Some of their consequences have already been discussed in several experimental conditions (17 and references therein). Let $S$ be given by:

$$
\begin{aligned}
S & =\int s\left(\omega_{L}-\omega_{0}\right) \mathrm{d} \omega_{L} \\
& =\int I(\tau) \mathrm{d}^{2} \stackrel{\circ}{N}
\end{aligned}
$$



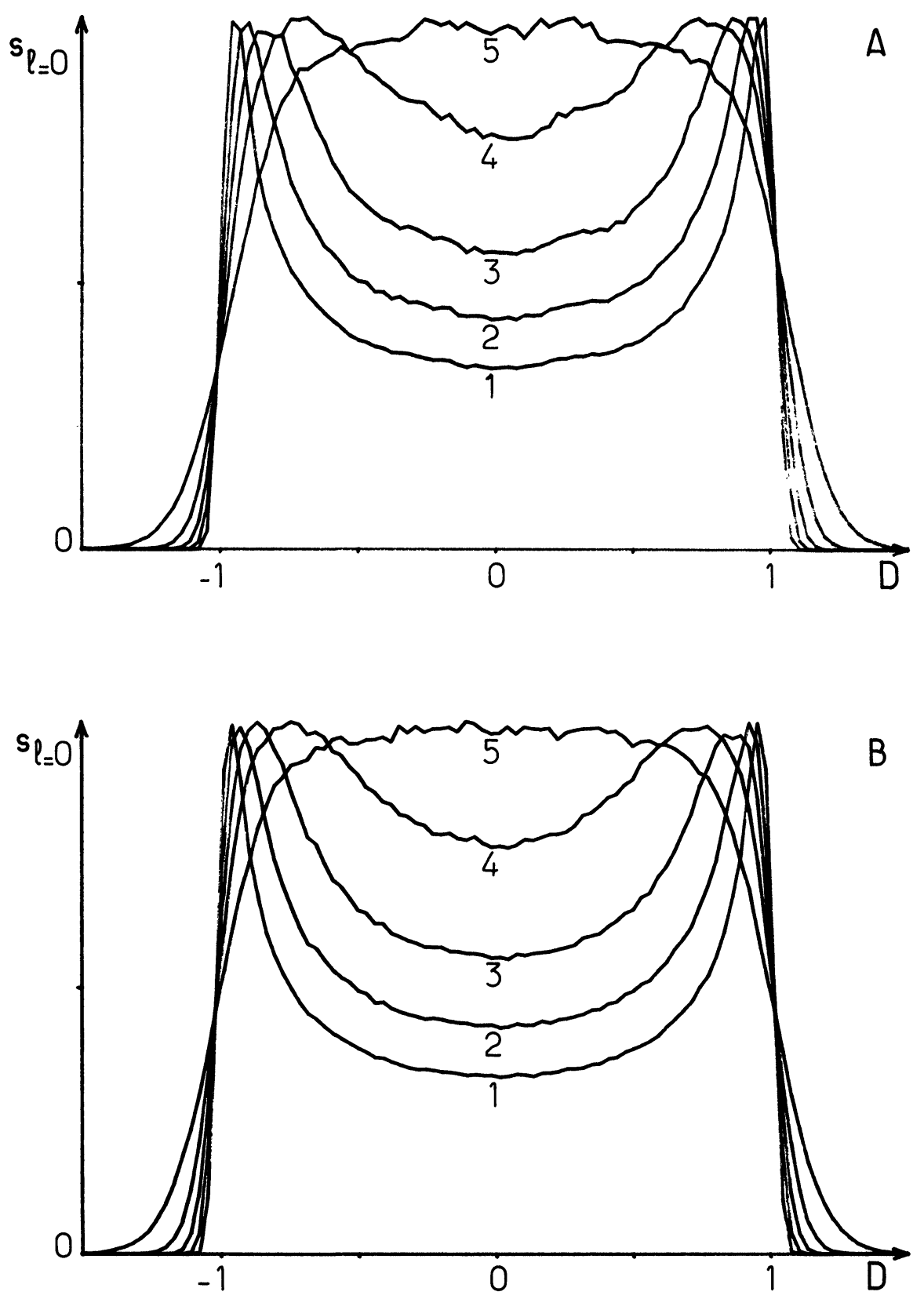

Figure 3 The $s_{l}\left(\omega_{L}-\omega_{0}\right)$ functions, with $l=0$, obtained for different values of the IF internal energy $E_{I F}$ (curves 1 to 5 correspond to $E_{I F}=0,5000,7500,9000$ and $10,000 \mathrm{~cm}^{-1}$ ), both in the ADDS (part a) and PADDS (part b) geometries. $s_{0}$ is plotted as a function of the dimensionless parameter $D=\left(\omega_{L}-\right.$ $\left.\omega_{0}\right) /\left(\omega_{0}\right) \times(c) /\left(\left\langle u_{I F}\right\rangle\right)$ where $\left\langle u_{I F}\right\rangle$ is the quadratic mean of the IF velocity in the centre of mass frame. Each calculation involves $2.510^{6}$ trajectories with a non zero interaction time and the $\mathrm{D}$ axis has been divided in 100 boxes. 

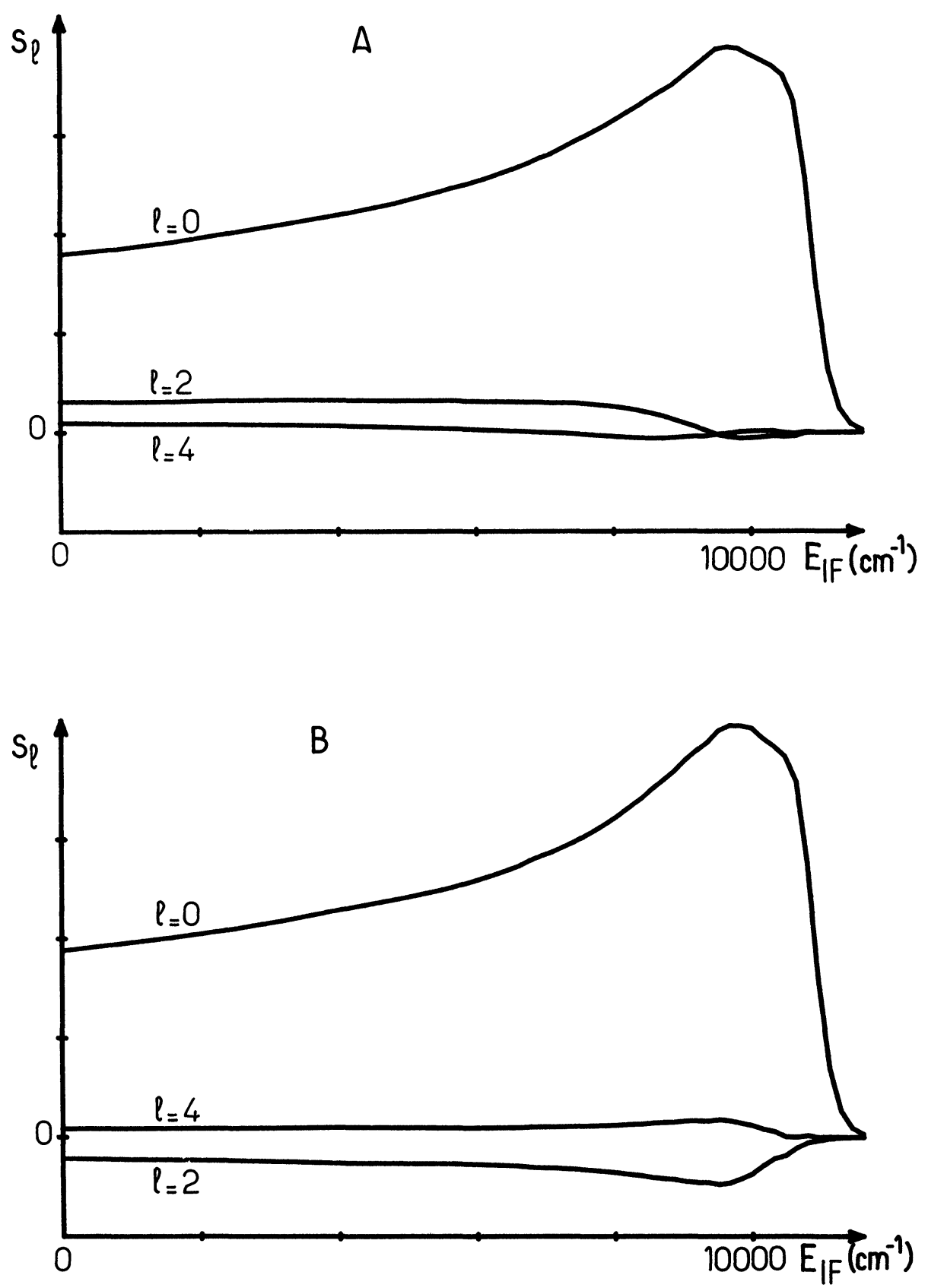

Figure $4 S_{l}$ coeefficients, measuring the sensitivity of the total LIF line intensity to the part of the differential cross section proportional to $P_{l}(\cos \theta)$, plotted for several $l$ values as a function of the IF internal energy, both for ADDS geometry (part a) and for PADDS geometry (part b). The mean total available energy is $10,886 \mathrm{~cm}^{-1}$. 
Developing $(\mathrm{d} \sigma / \mathrm{d} \Omega)$ on Legendre polynomials, we get:

$$
S=\sum_{l} \sigma_{l} S_{l}
$$

where $S_{l}=\int s_{l}\left(\omega_{L}-\omega_{0}\right) \mathrm{d} \omega_{L}$. Figure 4 shows the $S_{l}$ variations for different $l$ values, as a function of the IF internal energy $E_{I F}$, for both ADDS and PADDS geometries. Several comments can be done:

- The integrated line intensity $S$ is not only sensitive to the total cross section (= $4 \pi \sigma_{0}$ with our notations), but also to the shape of the differential cross section. $S$ is only sensitive to even order terms (for symmetry reasons) and, in practice, especially to $l=2$ as can be shown on Figure 4 . (The other $s_{l}$ functions oscillate too quickly, and they average out to zero). This means that, for a given integral cross section, the integrated line intensity depends on the sideways or backward/ forward character of the reaction.

- The sensitivity of the integrated line intensity to the total cross section is measured by the $S_{0}$ coefficient, which depends strongly on the IF internal energy. In the limiting case of large recoil velocity (case 18a), this sensitivity is proportional to:

$$
S_{0} \sim\left(\left\langle E_{a v}\right\rangle-E_{I F}\right)^{-1 / 2}
$$

where $\left(\left\langle E_{a v}\right\rangle-E_{I F}\right)$ is the mean recoil energy. (here $I(\tau) \sim \tau$ is assumed). Then for low IF internal energy, $S_{0}$ increases slowly with $E_{I F}$. This growth saturates when $u_{I F} \leqslant \mathrm{v}_{q}$. For higher $E_{I F}$ values only the most energetic collisions produce IF with the required internal energy, and the sensitivity $s_{0}$ goes down quickly.

Thus it appears clearly that neglecting the effects discussed here, and especially the dependence of the individual LIF signal $I(\tau)$ with the interaction time $\tau$, induces error in the measurement of the total cross section from the integrated LIF line intensity.

\section{CONCLUSION}

Since the work of Kinsey, ${ }_{1}^{1}$ it is well known that the Doppler profile of a laser induced detection line can be used to determine the differential cross section as a function of the product internal state. In the present paper we have tried to study the details of the relation between the Doppler profile and the differential cross section. We have made a coherent treatment of all the effects involved namely:

-the molecule laser interaction time depends on the recoil direction;

- the LIF signal is affected by saturation effects;

-the imperfections of a real experiment induce various averaging effects.

The calculations have been made with a Monte-Carlo integration. Their resuits hold qualitatively if the LIF signal depends on the molecule laser interaction time, being in a coherent saturation regime or not. They are going to be used to analyse our experimental data on the $F+I_{2} \rightarrow$ IF + I reaction. A rough preliminary analysis of 
this data ${ }^{18}$ has already shown a strong variation of the differential cross section with the rotational quantum number of IF, and we hope to get more accurate results with the detailed modelisation described here.

\section{References}

1. J. L. Kinsey, J. Chem. Phys. 66, 2560 (1977); W. D. Phillips, J. A. Serri, D. J. Ely, D. E. Pritchard, K. R. Way and J. L. Kinsey, Phys. Rev. Lett. 41, 937 (1978).

2. Several examples can be found in J. Chem. Soc. Faraday Trans. 2, 85 (1989).

3. E. J. Murphy, J. H. Brophy, G. S. Arnold, W. L. Dimpfl and J. L. Kinsey, J. Chem. Phys. 70, 5910 (1979); E. J. Murphy, J. H. Brophy and J. L. Kinsey, J. Chem. Phys. 74, 331 (1981); J. M. L'Hermite, G. Rahmat and R. Vetter, to be published in J. Chem. Phys.

4. B. Girard, N. Billy, G. Gouédard and J. Vigué, Chem. Phys. Lett. 136, 101 (1987).

5. J. A. Serri, J. L. Kinsey and D. E. Pritchard, J. Chem. Phys. 75, 663 (1981).

6. N. Billy, B. Girard, G. Gouédard and J. Vigué, Mol. Phys. 61, 65 (1987).

7. B. Girard, thèse d'Etat, Université P. et M. Curie (Paris VI), Paris, 1987.

8. M. Abramowitz and L. A. Stegun, Handbook of Mathematical functions, N.B.S. (1964).

9. H. Haberland, U. Buck and M. Tolle, Rev. Sci. Instrum. 56, 1712 (1985).

10. H. C. W. Beijerinck and N. F. Verster, Physica C111, 327 (1981).

11. G. M. McClelland, K. L. Saenger, J. J. Valentini and D. R. Herschbach, J. Phys. Chem. 83, 947 (1979).

12. E. Tiemann, J. Hoeft and T. Törring, Z. Naturforsch. 28a, 1405 (1973); H. D. Hartmann, H. Knöckel and E. Tiemann, Chem. Phys. Lett. 113, 364 (1985).

13. A. Omont, Prog. Quantum Electronics 5, 69 (1977).

14. N. W. Keane, J. C. Whitehead and R. Grice, J. Chem. Soc. Faraday Trans. 2, 85, 1081 (1989).

15. E. Luc-Koenig, C. Morillon and J. Verges, Physica 70, 175 (1973).

16. J. M. Hammersley and D. C. Handscomb, Monte-Carlo Methods, Wiley Ed. (1964).

17. C. Naulin, M. Costes, A. Bensedik and G. Dorthe, Laser Chem. 8, 283 (1988).

18. B. Girard, N. Billy, G. Gouédard and J. Vigué, J. Chem. Soc. Faraday Trans. 2 85, 1270 (1989). 